\title{
The Anti-proliferative activity of fuciodan on numerous cancer cell lines.
}

\author{
${ }^{1}$ Sadia Roshan, ${ }^{2}$ Amal Banafa, ${ }^{3}$ Yun-yi LIU (柳昀熠 ), ${ }^{4}$ Hui-jie CHEN ( 陈慧 \\ 洁), ${ }^{5}$ Guang-xiao YANG ( 杨广笑), ${ }^{6}$ Guang-yuan HE (何光源), \\ ${ }^{7}$ Ming-jie CHEN (陈明洁) ${ }^{\#}$
}

This work was supported by Wuhan Municipal Science and Technology Research Project (No. 201260523185) and the Public Science and Technology Research Funds Projects of Ocean (No. 201005013) College of Life Science and Technology, Huazhong University of Science \& Technology (HUST), Luoyu Road 1037, Wuhan, Hubei, 430074, P.R. China

Abstract: Fucoidan is one of the main bioactive components of polysaccharides. About 4percent of the total dry weight of many types of brown seaweed consists of polysaccharides known as fucoidan.It is a sulfated polysaccharide that possesses a complex structure. Chief components include a sulfuric esterified L-fucose, the trace elements of galactose,xylose and glucronic acid. The search for new drugs has raised interest in fucoidans. In the last few years, several fucoidans' structures have been solved. This review summarizes the research progress on the structure and bioactivity of fucoidansand the relationships between structure and bioactivity.

\section{Introduction}

Fucoidansare apoptosis-inducing polysaccharides which have significant percentages of L-fucose and sulfate ester groups. These are the structural unit of brown seaweed and some marine invertebrates such as sea urchins and sea cucumbers ${ }^{[1,2]}$.

Fucoidans isolated from different species have been extensively studied. They exhibit diverse biological activities such as antioxidant activity ${ }^{[3]}$, anti-inflammatoryactivity ${ }^{[4]}$, antiviral activity ${ }^{[5,}$, 6$]$ and antitumor activity ${ }^{[7]}$. Compared with other sulfated polysaccharides, fucoidans are widely available from different kinds of contemptible sources.So more and more fucoidans have been investigated in recent years and developed into the drugs or functional foods.

\section{Structure}

This polysachride was first isolated by Kylin from marine brown algae in 1913 and was named as "fucoidin". According to IUPAC rules, now it is termed as "fucoidan" but some called it fucan, fucosan or sulfated fucan.

At present fucoidan primed from Fucusvesiculosus is commercially available. It is composed of 44.1\%fucose, $26.3 \%$ sulfate, $31.1 \%$ ash and a little amino glucose whose $[\alpha] \mathrm{D}$ is $-123^{\circ[8,9]}$. Several marine algal polysaccharides, fucoidan in particular, have been found to induce apoptosis in cancer cells ${ }^{[10-12]}$. Recently, fucoidan has been reported to induce apoptosis in numerous cancer cell lines but the underlying mechanism is not elucidated yet because it is uncertain which cascade plays a pivotal role in the induction of apoptosis by fucoidan ${ }^{[13]}$.

\subsection{Fucoidans mainly composed of fucose and sulfate}

Fucoidan prepared from Fucusvesiculosusis commercially available at present.On the basis of the results of methylation and alkali treatment, Conchie and O'Neill found the main component unit was 1,2- $\alpha$ fucose and most of sulfate groups were located at position C-4 of the fucose units ${ }^{[14,15]}$. Anno et alisolated Lfucose 4-sulfate from it and the IR spectrum suggested that the sulfate group was substituted at the axial C-4 position of the L-fucospynanose ${ }^{[16]}$. The structural model of fucoidan of F. vesiculosussuggested by Conchie was accepted for forty years. In 1993, Pankteretalrevised this structural model suggesting that the core region of fucoidan was primarily a polymer of $\alpha-(1 \rightarrow 3)$ linked fucose with sulfate groups substituted at the C-4 position.Fucose was also attached to this polymer to form branched points, one for every 2-3 fucose residues within the chain (Figure 1).Pankter also explained the possible reasons for the different observations of Conchie. Firstly, the preparation methodwas different. Fucoidan analyzed in Conchie's studies was extracted with hot water.On the other hand acid extraction used by Pankter, has been the basis of the commercial preparation in recent years. Secondly, their methylation methods were different.Finally, Conchie analyzed the structure 
according to the chemical and chromatographic properties of the methylated products, and Pankter confirmed the methylated products by GC-EIMS ${ }^{[17]}$.

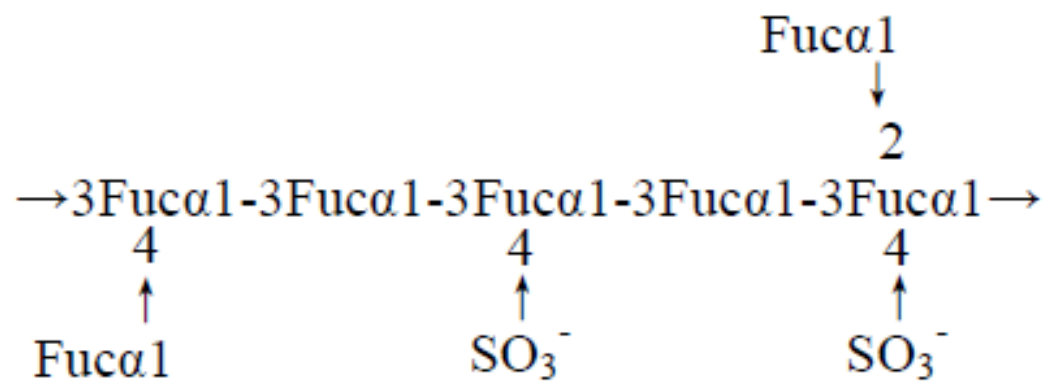

Fig.1 Chemical structure of fucoidan.

Bilanetalreported that fucoidans from the brown seaweeds $F$. evanescensC.Ag, F. distichusandF.serratusL.were consisted of fucose, sulfate and acetate $\left.{ }^{[2,} 18,19\right]$. Fucoidan of $F$. evanescensC.Ag.hasa linear backbone of alternating 3- and 4-linked $\alpha$-L-fucopyranose 2-sulfate residues: $\rightarrow 3$ )$\alpha$-L-Fucp (2SO3-)-(1 $\rightarrow 4)-\alpha$-L-Fucp(2SO3-)with additional sulfate occupying position 4 in apart of 3-linked fucose residues, whereas a part of the remaining hydroxyl groups was randomlyacetylated ${ }^{[2]}$. Fucoidan of $\mathrm{F}$. distichusis built up of disaccharide repeating units: $\rightarrow 3)-\alpha$-L-Fucp-(2, 4-di-SO3-)-(1 $\rightarrow 4)-\alpha-\mathrm{L}-\mathrm{Fuc} p$ $\left(2 \mathrm{SO}^{-}\right)$.The regular structure may be onlyslightly masked by random acetylation and undersulfation of several disaccharide repeating units ${ }^{[9]}$.Fucoidan from $F$. serratusL. has a branched structure, whose backbone is $\left.\rightarrow 3\right)-\alpha-$ L-Fucp-( $1 \rightarrow 4)-\alpha$-L-Fuc $p-(1 \rightarrow$, about half of the 3 -linked residues are substituted at C-4 by $\alpha$-L-Fuc $p$ - $(1 \rightarrow 4)-\alpha$ L-Fucp- $(1 \rightarrow 3)-\alpha$-L-Fuc $p$ - $(1 \rightarrow$ trifucoside units. Sulfate groups occupy mainly C-2 and sometimesC-4, although 3,4-diglycosylated and some terminal fucose residues may be nonsulfated. Acetategroups occupy C-4 of 3linked Fuc and C-3 of 4-linked Fuc in a ratio of about 7:3. The fucoidan alsocontains small amounts of xylose and galactose ${ }^{[19]}$. A sulfated fucan from Stoechospermummarginatumhas a backbone of $(1 \rightarrow 4)-$ and $(1 \rightarrow 3)-$ linked- $\alpha$-L-fucopyranosyl residues that aresubstituted at C-2 and C-3, and that fucosyl residues are sulfated mostly at C-2 and/or C- $4^{[20]}$. The ultrastructure of fucoidan can be studied using a variety of electron microscopy techniques.Sulfated fucan from Padinagymnosporaforms well-organized ultrastructures and exhibits particleswith polygonal forms with a polycrystalline structure. These particles are in fact constituted bysulfated fucan molecules since they are recognized by a lectin specific for $\alpha$-L-fucosyl residues. X-raymicroanalysis reveal that $\mathrm{S}$ is a constituent element, as expected for sulfated groups ${ }^{[21]}$.

\subsection{Fucoidans from other brown seaweeds}

The chemical composition of fucoidan from F. vesiculosusis comparatively simple.Other fucoidans have a compound composition. In 1962 Schweiger isolated a polysaccharide from Macrocytispyriferaand the ratio of fucose to galactose was 18:1.Schweiger first reported that fucoidan was not apurefucan sulfate but the heteropolymer of fucose, galactose and trace xylose ${ }^{[22]}$. Othersugars such as mannose, glucose, xylose and glucuronic acid (GlcA) had been found in fucoidansfromdifferent brown seaweeds (see Table 1), which increased the difficulty of structural analysis.

Table 1.Chemical compositions of some fucoidans

\begin{tabular}{|l|l|}
\hline Brown Seaweed & Chemical Composition \\
\hline F. vesiculosus & fucose, sulfate \\
\hline F. evanescens & fucose/sulfate/acetate $(1 / 1.23 / 0.36)$ \\
\cline { 2 - 2 } & \\
\hline F. distichus & fucose/sulfate/acetate $(1 / 1.21 / 0.08)$ \\
\cline { 2 - 2 } & \\
\hline F. serratusL & fucose/sulfate/acetate $(1 / 1 / 0.1)$ \\
\cline { 2 - 2 } & \\
\hline Lessoniavadosa & fucose/sulfate $(1 / 1.12)$ \\
\hline Pacrocytispyrifera & fucose/galactose $(18 / 1)$, sulfate \\
\hline Undariapinnatifida & fucose/galactose $(10 / 1)$, sulfate \\
\hline Ascophyllumnodosum & fucose/galactose $(1 / 1.1)$, sulfate \\
\hline HimanthalialoreaandBifurcaria bifurcate & fucose $(49 \%)$, xylose $(10 \%)$, GlcA $(11 \%)$ sulfate \\
\cline { 2 - 2 } & \\
\hline Padinapavonia & fucose, xylose, GlcA, sulfate \\
\hline
\end{tabular}


The Anti-proliferative activity of fuciodan on numerous cancer cell lines.

\begin{tabular}{|l|l|}
\hline & \\
\hline Laminariaangustata & fucose/galactose/sulfate \\
\hline Eckloniakurome & fucose, galactose, mannose, xylose, GlcA, sulfate \\
\cline { 2 - 2 } Sargassumstenophyllum & $\begin{array}{l}\text { fucose, galactose, mannose, GlcA, glucose, xylose, } \\
\text { sulfat }\end{array}$ \\
\hline Hizikiafusiforme & fucose, galactose, mannose, xylose, GlcA, sulfate \\
\cline { 2 - 2 } & \\
\hline Dictyotamenstrualis & \\
\hline Spatoglossumschroederi & fucose/xylose/galactose/sulfate \\
\hline
\end{tabular}

Numerous studies have shown that the chemical compositions and structures offucoidans from brown algae are very complex and their structures vary from species to species. Thedifferent backbone structures of fucoidans reflect the fundamental difference in fucoidans biosynthesis. In spite of numerous structural studies of algal fucoidans, their structure remainsunclear due to the absence of firm regularity, the presence of many minor components in some ofthem. These components are pentose, hexsoe, uronic acids, and sometimes protein component and random sulfation andacetylation. Sulfated fucan secluded from echinoderms have usually linear backbones and regularsulfation patterns resulting in the formation of oligosaccharide repeating units. The structures of theserepeating units can be determined unmistakably, especially by using high-field NMR spectroscopy,and hence, correlation between structures and biological action of polysaccharides may be outlined $^{[23,}{ }^{24]}$. Unfortunately, the structures of algal fucoidans are much more complicated. The algalpolysaccharides are usually heterogeneous and branched. Only partial information on theirstructures can be obtained by NMR spectroscopy. Controversial data may be found in the literature,even about the structure of the most carefully studied fucoidan from F. vesiculosus.

The same specific brown seaweed possibly possesses different structural fucoidans. Duarte et alreported that Sargassumstenophyllumbiosynthesized two different sets of fucoidans. One of them ischaracterized by higher percentages of GlcA and fewer sulfate groups, which are situated on differentsugar units. Fucose was the major component but other sugars like galactose, mannose, GlcA, glucoseand xylose were also in substantial amounts. Another fucoidan contains small amounts of GlcA andhigh percentages of sulfate groups, which are concentrated on the fucose residues, with only fucoseandgalactose as major components. Moreover the general basic structure of one fucoidan has a formalresemblance to that of the fucosylated chondroitin sulfates from the body wall of sea cucumbers, namely, a linear core (formed by $(1 \rightarrow 6)-\beta$-D-Gal and/or $(1 \rightarrow 2)-\beta-D-M a n$ units) with branched chainsof "fucan" (formed by $(1 \rightarrow 3)$ and/or $(1 \rightarrow 4)-\alpha$-L-Fuc, $(1 \rightarrow 4)$ - $\alpha$-D-GlcA, teminal $\beta$-D-Xyland,sometimes, $(1 \rightarrow 4)-\alpha$-D-Glu $)$.

Fucoidans extracted by different methods may have different structures. Ponce et al.reportedthat fucoidan of Adenocytutricularisextracted at room temperature was composed of mainly fucose,galactose and sulfate ester,the "galactofucan". The fucoidan extracted at $70^{\circ} \mathrm{C}$ was composed mainlyoffucose, accompanied by other monosaccharides (mostly mannose, but also glucose, xylose,rhamnose and galactose), significant amounts of uronic acids and low proportions of sulfate ester,namely "uronofucoidan".

\section{Biological Activities}

Fucoidan is a sulfated polysaccharidepurified from brown algae including Fucusand has a variety of biological effects including mobilization of hematopoietic progenitor cells.

The first recordedusesofherbsformedicaltreatmentbegan 4000 years ago. Andthistraditionaltreatment, originatingfrom China andIndia, spreadgraduallytoother countries. Recently, increasing attentionhasbeenfocusedontheapplication of natural products inlivercancertherapyallovertheworld ${ }^{[25]}$.

Yun-Young Byonet $a l^{[26]}$ reported that the sulfated polysaccharide fucoidan hasradioprotective effects on bone marrow cells (BMCs), which are the main cellular reservoir for the hematopoietic and immune vesiculoussystem.Fucoidan increased the viability of BMCs.Furthermorefucoidan altered the production of immune-related cytokines from BMCs and increased the capability of allogeeicsplenocytes.The result of this study facilitates the development of newradioprotective agents with reduced toxicity.

SuguruFukahori $e t a l^{[27]}$, examined the anti-tumor effects of fucoidan extracted from Okinawa mozuku on 15 human cancer cell lines (6 hepatocellular carcinomas, 1 cholangiocarcinoma, 1 gallbladder cancer, 2 ovarian cancers, 1 hepatoblastoma, 1 neuroblastoma and 3 renal cancers) using an MTT assay. Changes in apoptosis and the cell cycle were analyzed by flow cytometry. Cell proliferation was suppressed in 13 cell lines in a time- and/or dose-dependent manner. This suppression was marked in the hepatocellular carcinoma, cholangiocarcinoma and gallbladder carcinoma cell lines. In distinction, proliferation of the neuroblastoma and 1 of the 2 ovariancarcinoma cell lines was not affected. The ratio of apoptotic cells significantly increased in 5 of the 6 hepatocellular carcinoma cell lines, and the ratio of G2/M cells increased in the 3 hepatocellular cell 
lines examined. Their findings indicate that fucoidan is a potential anti-tumor agent for the treatment of bile duct cancers, such as hepatocellular carcinoma, cholangiocarcinoma and gall-bladder carcinoma. According to this study, Fucoidanis a potential anti-tumor medicine for specific cancers, such as HCC or cholangiocarcinoma. More detailed information on the anti-tumor effects of fucoidan should therefore be obtained in future animal studies.

Jae-Hee HYUN et $a l^{[28]}$, studied the antitumor activity of fucoidan from Fucusvesiculosusin HCT-15 colon carcinomaCell.AfterHCT-15 cells were treated with fucoidan, severalapoptotic events were observed, such as DNA fragmentation, chromatin condensation and increase of the population of sub-G1hypodiploid cells. In the mechanism of fucoidan-induced apoptosis, changes in Bcl-2and Bax protein expression levels and activation of caspases were observed. Fucoidan decreased Bcl-2 expression, whereas theexpression of Bax was increased in a time-dependent manner. In addition, the activeforms of caspase- 9 and caspase- 3 were increased, and the cleavage of poly(ADP-ribose) polymerase (PARP), avital substrate of effector caspase, was observed. Furthermore, the induction of apoptosis was also accompaniedby a strong activation of extracellular signalregulated kinase (ERK) and p38 kinase and an inactivation of phosphatidylinositol3-kinase (PI3K)/Akt in a time-dependent manner. These findings provide evidence demonstratingthat the pro-apoptotic effect of fucoidan is mediated through the activation of ERK, p38 and the blocking ofthe PI3K/Akt signal pathway in HCT-15 cells. These data support the hypothesis that fucoidan may have potentialin colon cancer treatment.

YoshinobuAisaet $a l^{[11]}$ reported that fucoidaninduces apoptosis of human HS-Sultancells accompanied by activation of Caspase-3 and down-regulation of ERK pathways. Fucoidan was found to inhibit proliferation and induce apoptosis in human lymphoma HS-Sultan cell lines. Fucoidan-induced apoptosis was accompanied by the activation of caspase- 3 and was partially prevented by pretreatment with a pan-caspase inhibitor, ZVAD-FMK. The mitochondrial potential in HS-Sultan cells was decreased $24 \mathrm{hr}$ after treatment with fucoidan, indicating that fucoidan induced apoptosis through a mitochondrial pathway. In contrast, phosphorylation of p38 and Akt was not altered by treatment with fucoidan. L-Selectin and P-selectin are known to be receptors of fucoidan; however, as HS-Sultan does not express either of these selectins, it is unlikely that fucoidan induced apoptosis through them in HS-Sultan. The neutralizing antibody, Dreg56, against human L-selectin did not prevent the inhibitory effect of fucoidan on the proliferation of IM9 and MOLT4 cells, both of which express Lselectin; thus it is possible fucoidan induced apoptosis though different receptors. These results demonstrate that fucoidan has direct anticancer effects on human HS-Sultan cells through caspase and ERK pathways.

TakeakiNagamineet $a l^{[29]}$ studied Inhibitory effect of fucoidan on Huh7 Hepatomacells through downregulation of CXCL12. The aim of this study was to assess whether fucoidan modulatesthe expression of chemokine ligand 12 (CXCL12)/chemokine receptor4 (CXCR4) and exerts antitumor activity toward Huh7hepatoma cells. According to MTT assays, fucoidan inhibitedthe growth of Huh7 cells and HepG2 cells in a dose-dependentmanner, with a 50\% inhibition of cell growth (IC50) of 2.0 and $4.0 \mathrm{mg} / \mathrm{ml}$, respectively. $\alpha$ fetoprotein levels in medium collectedfrom fucoidan-treated cells were significantly decreased in Huh7cells but not in HepG2 cells. Western blotting revealed that theamount of $\alpha$-fetoprotein was decreased by $1.0 \mathrm{mg} / \mathrm{ml} \mathrm{of}$ fucoidanin Huh7 cells, whereas it was unchanged in HepG2 cells. In Huh7cells, CXCL12 mRNA expression was significantly down-regulatedby $1.0 \mathrm{mg} / \mathrm{ml}$ of fucoidan, whereas CXCR4 mRNA expression wasunchanged by fucoidan. CXCL12 and CXCR4 mRNA were barelyexpressed inHepG2 cells. In addition, $1.0 \mathrm{mg} / \mathrm{ml} \mathrm{of}$ fucoidanmildlyarrested the cell cycle and induced apoptosis in Huh7 cells. Thefindings suggest that fucoidan exhibits antitumor activity towardHuh7 cells through the down-regulation of CXCL12 expression.

Hye-Jin Booet $a l^{[30]}$, studied that fucoidan from undariapinnatifida Induces apoptosis in A549 human lung carcinoma cells. The anticancer effects of fucoidan from Undariapinnatifida on A549 human lung carcinomacells were examined. Treatment of A549 cells with fucoidan resulted in potent antiproliferative activity. Also,some typical apoptotic characteristics, such as chromatin condensation and an increase in the population of sub-G1 hypodiploid cells, were observed. With respect to the mechanism underlying the induction of apoptosis, fucoidan reduced Bcl-2 expression, but the expression of Bax was increased in a dose-dependent mannercompared with the controls. Furthermore, fucoidan induced caspase- 9 activation, but decreased the level ofprocaspase-3. Cleavage of poly-ADP-ribose polymerase (PARP), a vital substrate of effector caspase, wasfound. The study further investigated the role of the MAPK and PI3K/Akt pathways with respect to theapoptotic effect of fucoidan, and showed that fucoidan activates ERK1/2 in A549 cells. Unlike ERK1/2,however, treatment with fucoidan resulted in the down-regulation of phosphor-p38 expression. In addition,fucoidan resulted in the down-regulation of phosphor-PI3K/Akt. Together, these results indicate that fucoidaninduces apoptosis of A549 human lung cancer cells through down-regulation of p38, PI3K/Akt, and theactivation of the ERK1/2 MAPK pathway.

YUMI YAMASAKI et $a l^{[31]}$, studied that fucoidaninduces apoptosis through activation of Caspase-8 on human Breast cancer MCF-7 Cells. Fucoidan is an active component of seaweed that has been shown to inhibit proliferation and induce apoptotic cell death in several tumor cells. In this report, the effect of fucoidan on the induction of apoptosis in human breast cancer MCF-7 cells was investigated. It demonstrated that 
fucoidan reduced the viable cell number of MCF-7 cells in a dose- and time-dependent manner. In contrast, fucoidan did not affect the viable cell number of normal human mammary epithelial cells. Results from the apoptosis assay demonstrated that fucoidan induced internucleosomal DNA fragmentation, chromatin condensation, activation of caspase- $7,-8$, and -9 , and cleavage of poly (ADP ribose) polymerase. Furthermore, expression of Bid was decreased, whereas truncated Bid was increased by fucoidan treatment. There was also a decline in cytosolic Bax and a striking increase of cytosolic cytochrome c. Caspase-8-specific inhibitor, z-ITEDfmk, canceled the cytotoxicity of fucoidan, activation of caspase- $7,-8$, and -9 , and a series of changes in Bax, Bid, and cytochrome c. However, caspase-9-specific inhibitor exerted a moderate inhibitory effect on the cytotoxicity of fucoidan. These data indicated that fucoidan could induce apoptotic cell death througha caspase8-dependent pathway in MCF-7 cells.

Kui-Jin Kim et al ${ }^{[32]}$, studied the repeated 4-week oral dose toxicity of fucoidan from the Sporophyll of Undariapinnatifida in Sprague-Dawley rats. Fucoidan is extracted from brown seaweeds, which can have anticoagulant, antithrombotic, antitumor, and antiviral activities. However, detailed studies on the toxicology of fucoidan have not been performed. In this study, the toxicity of fucoidan in Sprague-Dawleyratswastested. Fucoidan $(1350 \mathrm{mg} / \mathrm{kg}$ bw/day for 4 weeks) did not induce statistically significant differences in groups matched by gender with respect to body weight, ophthalmoscopy, urinalysis, hematology, and histopathology. Fucoidan did not change prothrombin time or activated partial thromboplastin time, indicating an inability to change blood clotting. This study demonstrated that fucoidan is not toxic under this administration paradigm.

Shinji Hayashi et al $^{[33]}$,studied that fucoidan partly prevents CCl4-induced liver fibrosis.In the present study, the effects of fucoidan on CCl4-induced liver fibrosis were investigated. Administration offucoidan reduced CCl4-induced acute and chronic liver failure. Hepatic fibrosis induced by $\mathrm{CCl} 4$ was also attenuated by injection of fucoidan. Damage to hepatocytes and activation of hepatic stellate cells are key events in liver fibrosis, and, interestingly, treatment of hepatocytes withfucoidan prevented CCl4-induced cell death and inhibited the proliferation hepatic stellate cells. These results indicate that fucoidan might be a promising antifibrotic agent possessing dual functions, namely, protection of hepatocytes and inhibition of hepatic stellate cell proliferation.

AndriySynytsyaet $a l^{[34]}$,studied the structure and antitumor activity of fucoidan isolated from sporophyllof Korean brown seaweed Undariapinnatifida.Fucoidan from the sporophyll (Miyeokgui) of cultured Korean brown seaweeds Undariapinnatifida(Miyeok) is interesting due to its various biological activities. This polysaccharide was isolated fromthe sporophyll (Miyeokgui) of Koreanseaweed U. pinatifida (Miyeok) was characterized by separation(GPC, CITP) and spectroscopic (FT-IR, FT-Raman, NMR) methods.

Taking into account the results obtained it may be concluded thatthis polysaccharide is sulphatedgalactofucan containing b-D-galactopyranoseand a-L-fucopyranose at near equal amounts $(44.6 \mathrm{~mol} \%$ and $50.9 \mathrm{~mol} \%)$. Xylose $(4.2 \mathrm{~mol} \%)$ and mannose $(0.3 \mathrm{~mol} \%)$ were found as minor sugars whileuronic acids were not detected.Fucoidan also contains significant amount of O-acetylgroups. Relationship between the galactan and fucan parts inwhole polysaccharide as well as the distribution of sulphate andacetate esters are unclear and need more investigation. Specificstructural properties of the Miyeokguifucoidan mentioned aboveas well as its evident antitumour activity comparable with thatof known biologically active commercial fucoidan from $\mathrm{F}$. vesiculosismake this polysaccharide interesting for medicinal use.

Marcel Tutor Aleet $a l^{[35]}$, studied thefucoidan from Sargassum sp. and Fucusvesiculosus reduces cell viability of lung carcinoma and melanoma cells in vitro and activates natural killer cells in mice in vivo. Fucoidan is known to exhibit crucial biological activities, including anti-tumor activity. In this study, the influence of crude fucoidan extracted from Sargassum sp. (MTA) and Fucusvesiculosus (SIG) on Lewis lung carcinoma cells (LCC) and melanoma B16 cells (MC) was examined. In vitro studies were performed using cell viability analysis and showed that SIG and MTA fucoidans significantly decreased the viable number of LCC and MC cells in a dose-response fashion. Histochemical staining showed morphological changes of melanoma B16 cells after exposure to fucoidan. The observed changes were indicative of crude fucoidan induced apoptosis. Male C57BL/6JJCL mice were subjected to daily i.p. injections over 4 days with either SIG or MTA fucoidan $(50 \mathrm{mg} / \mathrm{kg}$ body wt.). The cytolytic activity of natural killer (NK) cells was enhanced by crude fucoidan in a dose-dependent manner as indicated by $51 \mathrm{Cr}$ labeled YAC-1 target cell release.

This study provides substantial indications that crude fucoidan exerts bioactive effects on lung and skin cancer model cells in vitro and induces enhanced natural killer cell activity in mice in vivo. In this study the bioactivity of crude fucoidan through evaluation of its efficacy in controlling or inhibiting lung and skin cancer cell proliferation in vitro examined. The bioactivity of crude fucoidan towards these two types of cell lines was probably generated by the sulfate groups in the fucoidan structure. These findings need to be examined further to elucidate the underlying factors of fucoidan bioactivity. The study showed that crude fucoidan induces apoptosis of melanoma B16 cells and exerts anti-tumor activity through inhibition of the growth of Lewis lung carcinoma and melanoma B16 cells. In the present work, NK cells of mice treated with crude fucoidan acted as the principal effectors mediating tumor cell death. Overall, anti-tumor activity promoted by crude fucoidan was 
based on the enhancement of NK cell activity. Crude fucoidan from Sargassum sp. and F. vesiculosus thus appears to be a potent lung and skin cancer-preventive agent and its mode of action is associated with the immune response.

\section{Conclusions}

The fucoidans of brown algae are complex and heterogeneous. Their advanced structures have not been very clear. Their biological activities are so attractive that every year much research is being done on their structures and bioactivities. Because most biological activity studies are carried out using a relatively crude fucoidan preparation, it is not easy at present to determine the relationships between activity and structure. But it has become clear that at least some of these activities are not merely an effect of high charge density but have distinct structural specificities.

In conclusion fucoidanplays the anti-tumor or anti-proliferation role against human hepatoma cells etc. Due to the complicated metabolism of fucoidan in vivo and particularity of liver, further studies are necessary to determine the most suitable dose of fucoidan and drug delivery patches.In the animal experiments the nano technology and transcatheter hepatic arterial chemoembolization (TACE) may settle those problems and achieve more efficient cure of cancer in vivo.Future conformational studies of well-defined fucan structures should lead to better understanding of the biological properties of fucoidans.Deeply studying the structure of fucoidans and exploring the relationship activity and structure can provide theory foundation for developing and utilizing the brown algae resource.

\section{Reference}

[1]. Chizhov, A.O., et al., A study of fucoidan from the brown seaweed Chorda filum. Carbohydrate Research, 1999. 320(1-2): p. 108.

[2]. Bilan, M.I., et al., Structure of a fucoidan from the brown seaweed Fucus evanescens C. Ag. Carbohydrate Research, 2002. 337(8): p. 719.

[3]. Wang, J., et al., Antioxidant activity of sulfated polysaccharide fractions extracted from Laminaria japonica. International journal of biological macromolecules, 2008. 42(2): p. 127-132.

[4]. Matsumoto, S., et al., Fucoidan derived from Cladosiphon okamuranus Tokida ameliorates murine chronic colitis through the down?regulation of interleukin?6 production on colonic epithelial cells. Clinical \& Experimental Immunology, 2004. 136(3): p. 432-439.

[5]. Makarenkova, I., et al., [Inhibiting effects of fucoidans on Hantaan virus adsorption on a model of peritoneal macrophages in vitro]. Voprosy virusologii, 2008. 53(3): p. 12.

[6]. Hayashi, K., et al., Defensive effects of a fucoidan from brown alga Undaria pinnatifida against herpes simplex virus infection. International immunopharmacology, 2008. 8(1): p. 109-116.

[7]. Kwon, M.-J. and T.-J. Nam, Porphyran induces apoptosis related signal pathway in AGS gastric cancer cell lines. Life sciences, 2006. 79(20): p. 1956-1962.

[8]. Colliec, S., et al., Anticoagulant properties of a fucoidan fraction. Thrombosis research, 1991. 64(2): p. 143-154.

[9]. Nishino, T., et al., Isolation and partial characterization of a noval amino sugar-containing fucan sulfate from commercial Fucus vesiculosus fucoidan. Carbohydrate Research, 1994. 255: p. 213-224.

[10]. Sogawa, K., M. Matsuda, and K. Okutani, Induction of apoptosis by a marine microalgal polysaccharide in a human leukemic cell line. Journal of marine biotechnology, 1998. 6: p. 241-243.

[11]. Aisa, Y., et al., Fucoidan induces apoptosis of human HS?Sultan cells accompanied by activation of caspase? 3 and down regulation of ERK Pathways. American journal of hematology, 2005. 78(1): p. 7-14.

[12]. Teruya, T., et al., Anti-proliferative activity of oversulfated fucoidan from commercially cultured Cladosiphon okamuranus TOKIDA in U937 cells. International journal of biological macromolecules, 2007. 41(3): p. 221-226.

[13]. Philchenkov, A., et al., Sensitization of human malignant lymphoid cells to etoposide by fucoidan, a brown seaweed polysaccharide. Exp. Oncol, 2007. 29: p. 181-185.

[14]. Conchie, J. and E. Percival, 167. Fucoidin. Part II. The hydrolysis of a methylated fucoidin prepared from Fucus vesiculosus. Journal of the Chemical Society (Resumed), 1950: p. 827-832.

[15]. O'Neil, A., Degradative studies on fucoidan. Journal of American Chemical Society, 1954. 76(20): p. 5074-5076.

[16]. Anno, K., N. Seno, and M. Ota, Isolation of L-fucose 4-sulfate from fucoidan. Carbohydrate Research, 1970. 13(1): p. 167-169.

[17]. Patankar, M.S., et al., A revised structure for fucoidan may explain some of its biological activities. Journal of Biological Chemistry, 1993. 268(29): p. 21770-21776.

[18]. Bilan, M.I., et al., A highly regular fraction of a fucoidan from the brown seaweed Fucus distichus $L$. Carbohydrate Research, 2004. 339(3): p. 511.

[19]. Bilan, M.I., et al., Structure of a fucoidan from the brown seaweed Fucus serratus L. Carbohydrate Research, 2006. $341(2)$ : p. 238.

[20]. Adhikari, U., et al., Structure and antiviral activity of sulfated fucans from Stoechospermum marginatum. Phytochemistry, 2006. 67(22): p. 2474-2482.

[21]. Andrade, L.R., et al., Ultrastructure of acidic polysaccharides from the cell walls of brown algae. Journal of structural biology, 2004. 145(3): p. 216-225.

[22]. Ji, M., Algae chemistry. 1997, Science Publishing House: Beijing.

[23]. Ribeiro, A.-C., et al., A sulfated -L-fucan from sea cucumber. Carbohydrate Research, 1994. 255: p. 225-240.

[24]. PavÃ £o, M.S., P.A. Mour, and B. Mulloy, Structure of a unique sulfated -galactofucan from the tunicate Clavelina. Carbohydrate Research, 1990. 208: p. 153-161.

[25]. Kaefer, C.M. and J.A. Milner, The role of herbs and spices in cancer prevention. The Journal of nutritional biochemistry, 2008. 19(6): p. 347-361.

[26]. Byon, Y.-Y., et al., Radioprotective effects of fucoidan on bone marrow cells: improvement of the cell survival and immunoreactivity. Journal of veterinary science, 2008. 9(4): p. 359-365.

[27]. Fukahori, S., et al., Fucoidan, a major component of brown seaweed, prohibits the growth of human cancer cell lines in vitro. Mol. Med. Report, 2008. 1: p. 537-542. 
[28]. Hyun, J.-H., et al., Apoptosis inducing activity of fucoidan in HCT-15 colon carcinoma cells. Biological and Pharmaceutical Bulletin, 2009. 32(10): p. 1760-1764.

[29]. Nagamine, T., et al., Inhibitory effect of fucoidan on Huh7 hepatoma cells through downregulation of CXCL12. Nutrition and cancer, 2009. 61(3): p. 340-347.

[30]. Boo, H.J., et al., Fucoidan from Undaria pinnatifida induces apoptosis in A549 human lung carcinoma cells. Phytotherapy Research. 25(7): p. 1082-1086.

[31]. Yamasaki-Miyamoto, Y., et al., Fucoidan induces apoptosis through activation of caspase-8 on human breast cancer MCF-7 cells. Journal of agricultural and food chemistry, 2009. 57(18): p. 8677-8682.

[32]. Kim, K.-J., et al., A 4-week repeated oral dose toxicity study of fucoidan from the Sporophyll of Undaria pinnatifida in Sprague Dawley rats. Toxicology. 267(1): p. 154-158.

[33]. Hayashi, S., et al., Fucoidan partly prevents CCl 4-induced liver fibrosis. European journal of pharmacology, 2008. 580(3): p. 380384.

[34]. Synytsya, A., et al., Structure and antitumour activity of fucoidan isolated from sporophyll of Korean brown seaweed Undaria pinnatifida. Carbohydrate Polymers. 81(1): p. 41-48.

[35]. Ale, M.T., et al., Fucoidan from Sargassum sp. andFucus vesiculosus reduces cell viability of lung carcinoma and melanoma cells in vitro and activates natural killer cells in mice in vivo. International journal of biological macromolecules. 49(3): p. 331-336. 